\title{
A note on the Kazdan-Warner type condition
}

\author{
by
}

\author{
Zheng-chao HAN and YanYan LI*
}

Department of Mathematics, Rutgers University, New Brunswick, NJ 08903 USA

ABSTRACT. - This paper addresses the necessary conditions for a function $K(x)$ on $\mathbf{S}^{n}$ to be the scalar curvature function of a metric pointwise conformal to the standard metric on $\mathbf{S}^{n}$. The well known necessary conditions are: $K(x)$ is positive somewhere and satisfies the Kazdan-Warner type condition (see the text below). It has remained an outstanding problem for many years whether the above necessary conditions are also sufficient. Recently W. Chen and $\mathrm{C}$. $\mathrm{Li}$ ([ChL]) proved that the above conditions are not sufficient by producing changing sign functions $K$ which satisfy the above conditions, but are not scalar curvature functions of any metric pointwise conformal to the standard metric on $\mathbf{S}^{n}$. In their construction, it is essential that $K$ changes sign. In fact, for $n=2$, it follows from the results of $[\mathrm{XY}]$ that for the class of positive nondegenerate axisymmetric functions the Kazdan-Warner type condition is actually necessary and sufficient. This brings up a natural question that whether this is a general fact, or it is only so for axisymmetric functions. In this note we answer the above question for $2 \leq n \leq 4$ by producing a family of positive functions $K$ which satisfy the Kazdan-Warner type condition, but nevertheless are not scalar curvature functions of any metric pointwise conformal to the standard metric on $\mathbf{S}^{n}$.

RÉSUMÉ. - Nous construisons certaines fonctions positives sur $\mathbf{S}^{n}$, $2 \leq n \leq 4$, qui satisfont les conditions de type Kazdan-Warner, mais telles qu'elles ne sont pas les courbures scalaires des métriques conformes à la métrique standard de $\mathbf{S}^{n}$.

* Partially supported by the Alfred P. Sloan foundation Research Fellowship and an NSF grant. 


\section{INTRODUCTION}

Let $\left(\mathbf{S}^{n}, g_{0}\right)$ be the standard $n$-sphere. The following question was raised by Professor L. Nirenberg: which function $K(x)$ on $\mathbf{S}^{2}$ is the Gauss curvature of a metric $g$ on $\mathbf{S}^{2}$ pointwise conformally equivalent to $g_{0}$ ? Naturally one may ask a similar question in higher dimensional case, namely, which function $K(x)$ on $\mathbf{S}^{n}$ is the scalar curvature of a metric $g$ on $\mathbf{S}^{n}$ conformally equivalent to $g_{0}$ ?

For $n=2$, if we write $g=e^{2 v} g_{0}$, the problem is equivalent to finding a function $v$ on $\mathbf{S}^{2}$ which satisfies the following equation

$$
-\Delta_{g_{0}} v+1=K(x) e^{2 v} .
$$

For $n \geq 3$, if we write $g=v^{\frac{4}{n-2}} g_{0}$, the problem is equivalent to finding a function $v$ on $\mathbf{S}^{n}$ which satisfies the following equation

$$
-\Delta_{g_{0}} v+c(n) R_{0} v=c(n) K(x) v^{\frac{n+2}{n-2}}, \quad v>0,
$$

where $c(n)=\frac{n-2}{4(n-1)}, R_{0}=n(n-1)$ is the scalar curvature of $g_{0}$ and $\Delta_{g_{0}}$ denotes the Laplace-Beltrami operator associated with the metric $g_{0}$.

A necessary condition for solving (1) or (2) is that $K$ be positive somewhere. For $n=2$, this follows from integrating (1) on $\mathbf{S}^{2}$. For $n \geq 3$, this follows from multiplying (2) by $v$ and integrating by parts on $\mathbf{S}^{n}$. Kazdan and Warner discovered in [KW] another much more significant condition by exploiting centered dilation conformal transformations of $\mathbf{S}^{n}$ which asserts that a solution to the problem should satisfy

$$
\int_{\mathbf{S}^{n}} X(K) d V_{g}=0
$$

for $X$ being gradients of first order spherical harmonics. Here $d V_{g}=$ $e^{2 v} d V_{g_{0}}$ for $n=2$ and $d V_{g}=v^{\frac{2 n}{n-2}} d V_{g_{0}}$ for $n \geq 3, d V_{g_{0}}$ is the volume element of $g_{0}$. Bourguignon and Ezin generalized it in [BE] by exploiting the full conformal transformation group of $\mathbf{S}^{n}$ which asserts that a solution to the problem should satisfy

$$
\int_{\mathbf{S}^{n}} X(K) d V_{g}=0
$$

for any conformal Killing vector field $X$ of $\mathbf{S}^{n}$. We recall that a vector field $X$ on $\mathbf{S}^{n}$ is called a conformal Killing vector field if there exists a 
one parameter family of conformal diffeomorphisms $\varphi_{t}(-\epsilon<t<\epsilon)$ of $\mathbf{S}^{n}$ such that $\varphi_{0}=$ identity and $X=\left.\frac{d}{d t} \varphi_{t}\right|_{t=0}$.

In the following we say that a function $K$ on $\mathbf{S}^{n}$ satisfies the KazdanWarner type condition if there exists some positive function $f$ on $\mathbf{S}^{n}$ satisfying

$$
\int_{\mathbf{S}^{n}} X(K) f d V_{g_{0}}=0,
$$

for any conformal Killing vector field $X$ of $\mathbf{S}^{n}$.

For convenience we introduce stereographic projection coordinates of $\mathbf{S}^{n}$. Let $P$ be the south pole and we make a stereographic projection to the equatorial plane of $\mathbf{S}^{n}$. Let $x=\left(x_{1}, x_{2}, \cdots, x_{n+1}\right) \in \mathbf{S}^{n}$ and let $y=\left(y_{1}, \cdots, y_{n}\right) \in \mathbf{R}^{n}$ denote the stereographic projection coordinates of $x$. It is easy to see that

$$
\begin{cases}x_{i}=\frac{2 y_{i}}{1+|y|^{2}}, & 1 \leq i \leq n, \\ x_{n+1}=\frac{|y|^{2}-1}{|y|^{2}+1}, & \\ y_{i}=\frac{x_{i}}{1-x_{n+1}}, & 1 \leq i \leq n,\end{cases}
$$

and

$$
g_{0}=\left(\frac{2}{1+y^{2}}\right)^{2} d y^{2}
$$

The set of all conformal Killing vector fields of $\mathbf{S}^{n}$ forms a linear space of dimension $(n+1)(n+2) / 2$ and $\left\{X_{i} \mid 1 \leq i \leq(n+1)(n+2) / 2\right\}$ forms a basis, where $X_{i}=\nabla_{g_{0}} x_{i}$ for $1 \leq i \leq n+1$ and $X_{i}$ are generators of rotations for $n+2 \leq i \leq(n+1)(n+2) / 2$.

We know that if $K$ does not satisfy the Kazdan-Warner type condition then there is no solution to (1) $(n=2)$ or $(2)(n \geq 3)$. It is natural to ask whether or not $(1)(n=2)$ or $(2)(n \geq 3)$ has a solution for any function $K$ on $\mathbf{S}^{n}$ which is positive somewhere and satisfies the KazdanWarner type condition. Extensive study has been made and various existence results have been obtained (see the references in the end and the references therein). Nevertheless the above question had been left open for quite some time. Some interesting results have been obtained for axisymmetric functions $K$. We say that a function $K$ is axisymmetric if $K=K(\theta)$ where $\cos \theta=x_{n+1}$. For $n=2, \mathrm{X}$. Xu and P. Yang constructed in [XY] some changing sign axisymmetric functions $K$ and proved that they satisfy the Kazdan-Warner type condition but (1) has no axisymmetric solution. 
For $n \geq 3$, Bianchi and Engnell constructed some positive axisymmetric functions $K$ and proved that they satisfy the Kazdan-Warner type condition but (2) has no axisymmetric solution. However they did not know whether or not (1) $(n=2)$ or (2) $(n \geq 3)$ has a solution.

In a recent paper [ChL] Chen and Li proved that for an axisymmetric function $K(\theta)(1)(n=2)$ and (2) $(n \geq 3)$ have no solution at all if $K(\theta)$ is monotone in the region where $K$ is positive. In particular, there are many changing sign functions $K$ on $\mathbf{S}^{n}(n \geq 2)$ satisfying the Kazdan-Warner type condition, but (1) $(n=2)$ and (2) $(n \geq 3)$ have no solution. In their construction, it is essential that $K$ changes sign. In fact it follows from the results of [XY] that for the class of positive nondegenerate axisymmetric functions the Kazdan-Warner type condition is actually necessary and sufficient for $(1)(n=2)$ to have a solution. This brings up a natural question that whether this is a general fact, or it is only so for axisymmetric functions.

In this note we answer the above question by producing some positive functions $K$ which satisfy the Kazdan-Warner type condition, but nevertheless (1) $(n=2)$ and (2) $(n=3,4)$ have no solution. The basic idea for constructing these functions is the following. Using some compactness results for solutions of (1) $(n=2)$ and (2) $(n=3,4)$, we produce a family of functions $K$ for which (1) $(n=2)$ and (2) $(n=3,4)$ have no solutions. We then show that these functions satisfy the Kazdan-Warner type condition. There are actually many such functions, but for simplicity we only present some with certain symmetry of $\mathbf{S}^{n}$.

\section{STATEMENT AND PROOF OF THE RESULT}

Let $\mathcal{F}_{1}$ denote the set of functions $h$ on $\mathbf{S}^{n}$ which satisfy

$$
\begin{gathered}
h\left(\cdots,-x_{i}, \cdots\right)=h\left(\cdots, x_{i}, \cdots\right), \\
\forall\left(x_{1}, \cdots, x_{n+1}\right) \in \mathbf{S}^{n}, \quad 1 \leq i \leq n, \\
h\left(x_{1}, \cdots, x_{n},-x_{n+1}\right)=-h\left(x_{1}, \cdots, x_{n}, x_{n+1}\right), \quad \forall\left(x_{1}, \cdots, x_{n+1}\right) \in \mathbf{S}^{n},
\end{gathered}
$$

and $\mathcal{F}_{2}$ denote the set of functions $f$ on $\mathbf{S}^{n}$ which satisfy

$$
\begin{gathered}
f\left(\cdots,-x_{i}, \cdots, x_{n+1}\right)=f\left(\cdots, x_{i}, \cdots, x_{n+1}\right), \\
\forall\left(x_{1}, \cdots, x_{n+1}\right) \in \mathbf{S}^{n}, \quad 1 \leq i \leq n+1 .
\end{gathered}
$$

Proposition 1. - For $n \geq 2, h \in \mathcal{F}_{1}, f \in \mathcal{F}_{2}$, we have

$$
\begin{gathered}
\int_{\mathbf{S}^{n}} X_{i}(h) f d V_{g_{0}}=0, \\
\forall 1 \leq i \leq n, \text { and } n+2 \leq i \leq(n+1)(n+2) / 2 .
\end{gathered}
$$


Proof. - For $h \in \mathcal{F}_{1}$, it is easy to see that $X_{i}(h)\left(\cdots,-x_{i}, \cdots\right)=$ $-X_{i}(h)\left(\cdots, x_{i}, \cdots\right), \forall x \in \mathbf{S}^{n}, 1 \leq i \leq n$, and $X_{i}(h)(-x)=$ $-X_{i}(h)(x), \forall x \in \mathbf{S}^{n}, n+2 \leq i \leq(n+1)(n+2) / 2$. (5) follows immediately.

First we fix $\delta>0$ small and construct a function $h_{0} \in C^{\infty}\left(\mathbf{S}^{n}\right) \cap \mathcal{F}_{1}$ with the following properties:

$$
\begin{gathered}
h_{0}(x)=\left\{\begin{array}{r}
-1+y_{1}^{2}+y_{2}^{4}, \quad\left|y_{1}\right|^{2}+\cdots+\left|y_{n}\right|^{2} \leq \delta, \\
x_{n+1}, \quad \sqrt{\delta} \leq\left|y_{1}\right|^{2}+\cdots+\left|y_{n}\right|^{2} \leq 1,
\end{array}\right. \\
\left|h_{0}(x)\right|<3 / 2, \quad \delta \leq\left|y_{1}\right|^{2}+\cdots+\left|y_{n}\right|^{2} \leq \sqrt{\delta}, \\
h_{0}\left(\cdots,-x_{i}, \cdots\right)=h_{0}\left(\cdots, x_{i}, \cdots\right), \quad \forall x \in \mathbf{S}^{n}, x_{n+1} \leq 0,1 \leq i \leq n, \\
h_{0}\left(x_{1}, \cdots, x_{n}, x_{n+1}\right)=-h_{0}\left(x_{1}, \cdots, x_{n},-x_{n+1}\right), \quad \forall x \in \mathbf{S}^{n}, x_{n+1} \geq 0, \\
\nabla_{g_{0}} x_{n+1}(x) \cdot \nabla_{g_{0}} h_{0}(x) \geq 0 \quad \forall x \in \mathbf{S}^{n},
\end{gathered}
$$

and the equality holds if and only if $x=(0, \cdots, 0, \pm 1)$.

The construction of $h_{0}$ is elementary and we leave it to the readers.

Now for $0<\epsilon<<\delta$, we construct a family of functions $\left\{h_{\epsilon}\right\} \in$ $C^{\infty}\left(\mathbf{S}^{n}\right) \cap \mathcal{F}_{1}$ satisfying the following properties:

$$
\begin{gathered}
h_{\epsilon}(x)=\left\{\begin{aligned}
-1+y_{1}^{2}-\epsilon y_{2}^{2}+y_{2}^{4}, & \left|y_{1}\right|^{2}+\cdots+\left|y_{n}\right|^{2} \leq \delta^{3}, \\
h_{0}(x), & \delta^{2} \leq\left|y_{1}\right|^{2}+\cdots+\left|y_{n}\right|^{2} \leq 1,
\end{aligned}\right. \\
h_{\epsilon}\left(\cdots,-x_{i}, \cdots\right)=h_{\epsilon}\left(\cdots, x_{i}, \cdots\right), \quad \forall x \in \mathbf{S}^{n}, x_{n+1} \leq 0,1 \leq i \leq n, \\
h_{\epsilon}\left(x_{1}, \cdots, x_{n}, x_{n+1}\right)=-h_{\epsilon}\left(x_{1}, \cdots, x_{n},-x_{n+1}\right), \quad \forall x \in \mathbf{S}^{n}, x_{n+1} \geq 0, \\
\left|\nabla_{g_{0}} h_{\epsilon}(x)\right| \geq 1 / C(\delta, n)>0, \quad \delta^{3} \leq\left|y_{1}\right|^{2}+\cdots+\left|y_{n}\right|^{2} \leq \delta, \\
h_{\epsilon} \rightarrow h_{0} \quad \text { in } C^{\infty}\left(\mathbf{S}^{n}\right) \text { as } \epsilon \text { goes to } 0 .
\end{gathered}
$$

Set

$$
K_{0}=2+h_{0}, \quad \text { and } \quad K_{\epsilon}=2+h_{\epsilon} .
$$

Obviously $1 / 2 \leq K_{\epsilon} \leq 5 / 2$ for $\epsilon$ small and $K_{0}$ does not satisfy the Kazdan-Warner type condition.

Proposition 2. - For $2 \leq n \leq 4$ and $\epsilon>0$ small enough, there exists some smooth positive function $f_{\epsilon} \in \mathcal{F}_{2}$ such that

$$
\int_{\mathbf{S}^{n}} X_{i}\left(K_{\epsilon}\right) f_{\epsilon} d V_{g_{0}}=0, \quad \forall 1 \leq i \leq(n+1)(n+2) / 2 .
$$


Proof of Proposition 2. - It is elementary to see that $X_{n+1}\left(K_{\epsilon}\right) \in \mathcal{F}_{2}$ and near the south pole ( $y$ close to zero)

$$
X_{n+1}\left(K_{\epsilon}\right)(y)=8 y_{1}^{2}-8 \epsilon y_{2}^{2}+O\left(|y|^{4}\right)
$$

It follows that $X_{n+1}\left(K_{\epsilon}\right)$ changes sign and therefore there exists some smooth positive function $f_{\epsilon} \in \mathcal{F}_{2}$, such that, (6) holds for $i=n+1$. For $1 \leq i \leq n$ and $n+2 \leq i \leq(n+1)(n+2) / 2$, (6) follows from Proposition 1.

The following is our main result.

THEOREM. - For $2 \leq n \leq 4$ and $\epsilon>0$ small enough, (1) for $n=2$ and (2) for $n=3,4$ have no solution for $K=K_{\epsilon}$.

The above Theorem and Proposition 2 show that for $\epsilon>0$ small enough, $K_{\epsilon}$ are positive smooth functions on $\mathbf{S}^{n}(2 \leq n \leq 4)$ which satisfy the Kazdan-Warner type condition, but nevertheless (1) $(n=2)$ and (2) ( $n=3,4)$ have no solution at all. The following is a proof of the Theorem, modulo the statements of the relevant compactness results, for which we refer to the Appendix.

Proof of Theorem. - As noted earlier, $K_{0}$ does not satisfy the KazdanWarner type condition, so (1) $(n=2)$ and (2) $(n=3,4)$ have no solution for $K_{0}$. By the compactness results (for $n=2,3$, see Theorem $\mathrm{A}$ in the Appendix; for $n=4$, see Theorem 0.8 in [L3]), (1) $(n=2)$ and (2) ( $n=3,4$ ) have no solution for $K_{\epsilon}$ for all $\epsilon>0$ small. In fact for all $K$ lying in a $C^{2}$ neighborhood of $K_{0},(1)(n=2)$ and (2) $(n=3,4)$ have no solution. This proves our Theorem.

\section{APPENDIX}

We first state the compactness results that we need for solutions of (1) $(n=2)$ and solutions of (2) $(n=3)$.

Theorem A. - For $n=2,3$, let $K \in C^{2}\left(\mathbf{S}^{n}\right)$ satisfy for some constants $K_{1}, d>0$ that

$$
K_{1} \leq K(x), \quad \forall x \in \mathbf{S}^{n}
$$

$$
\min _{\left\{x \in \mathbf{S}^{n},|\nabla K(x)| \leq d\right\}}\left|\Delta_{g_{0}} K(x)\right| \geq d .
$$


Then for all solutions $v$ of (1) for $n=2$ and (2) for $n=3,0<\alpha<1$,

$$
\|v\|_{C^{3, \alpha}\left(\mathbf{S}^{n}\right)} \leq C \text {. }
$$

Where $C$ depends only on $K_{1}, d,\|K\|_{C^{2}\left(\mathbf{S}^{n}\right)}, \alpha$ and the modulo of continuity of $\nabla_{g_{0}}^{2} K$ on $\mathbf{S}^{n}$.

We remark that, for $n=3$, it follows from (8) and standard elliptic theories ([GT]), $\left\|v^{-1}\right\|_{C^{3, \alpha}\left(\mathbf{S}^{3}\right)} \leq C$ for a solution $v$ of (2).

For $n=2$, Theorem A was established in [H] (Theorem 3 there) and in [CGY] (Theorem 2 there). For $n=3$, Theorem A was established in [L1] (Theorem 0.4 there) based on [Sc2], [BC], and was established in [CGY] (Theorem 2 there) by somewhat different approach. Certain compactness results of similar type for $n \geq 4$ have been established by the second author more recently. See [L2-3] for details.

For readers' convenience, we give some ideas of a proof of Theorem A.

Idea of a proof of Theorem A. - Let us take this opportunity to clarify the meaning of Theorem 3 in $[\mathrm{H}]$. For a fixed $K>0$ on $\mathbf{S}^{2}$ satisfying the conditions listed in Theorem 3 of $[\mathrm{H}]$, there are apriori estimates for all solutions of (1). In order to obtain uniform estimates for solutions of (1) for a family of $K^{\prime}$ s, condition (7) above should replace the lower bound condition for $|\Delta K|$ at the critical points of $K$. This remark seems to apply also to Theorem 2 in [CGY]. The proof in [H] essentially works to prove Theorem A (there were some errors in the proof of Theorem 3 in $[\mathrm{H}]$ on the line below equation (20) and the fifth line below equation (20) on p. 700 of $[\mathrm{H}]$, caused by oversimplification in the process of transcribing the manuscript into typing. The following sketch is the original correct version). It is easy to see that we only need to estimate $\max _{\mathbf{S}^{2}} v$. Suppose, on the contrary, that there exist a sequence of $K_{j}$ satisfying the conditions in Theorem $\mathrm{A}$, and corresponding solutions $v_{j}$ such that $\max v_{j} \rightarrow \infty$. Let $Q_{j}$ be such that $v_{j}\left(Q_{j}\right)=\max v_{j}$, and $t_{j}=\sqrt{K_{j}\left(Q_{j}\right)} e^{v_{j}\left(Q_{j}\right)}$. For each $j$, we use the stereographic projection coordinates with $Q_{j}$ as the south pole to define

$$
\begin{aligned}
u_{j}(y) & =v_{j}\left(\frac{y}{t_{j}}\right)+\log \frac{t_{j}\left(1+|y|^{2}\right)}{1+|y|^{2} / t_{j}^{2}}+\frac{1}{2} \log K_{j}\left(Q_{j}\right) \\
& =v_{j}\left(\frac{y}{t_{j}}\right)+\log \frac{1+|y|^{2}}{1+|y|^{2} / t_{j}^{2}}-v_{j}\left(Q_{j}\right),
\end{aligned}
$$

here and in the following we will use a point on $\mathbf{S}^{2}$ and its appropriate stereographic coordinates interchangeably. Then $u_{j}$ satisfies

$$
\Delta u_{j}=1-\frac{K_{j}\left(y / t_{j}\right)}{K_{j}\left(Q_{j}\right)} e^{2 u_{j}} .
$$

Vol. 13, $\mathrm{n}^{\circ} 3-1996$. 
From our choice of $Q_{j}, t_{j}$, we have $u_{j}\left(Q_{j}\right)=0$ and $u_{j}(y) \leq$ $\log \frac{1+|y|^{2}}{1+|y|^{2} / t_{j}^{2}}$. The corresponding formulas in [H] were computed using the same coordinates as here. But some errors occurred when they were translated into the abstract notation there. If, after subtracting a subsequence, $Q_{j} \rightarrow Q$, then elliptic theory implies that, away from $-Q, u_{j}$ converges uniformly to a solution $u$ of

$$
\Delta u=1-e^{2 u}, u(Q)=0, u(y) \leq \log \left(1+|y|^{2}\right), \int_{\mathbf{S}^{2}} e^{2 u} \leq 4 \pi .
$$

The last inequality follows from the Gauss-Bonnet theorem and the Fatou's lemma. From the classification of solutions of the above equation, we know that $u \equiv 0$. To conclude global uniform convergence, we note that convergence of $u_{j}$ to $u$ away from $-Q$ and the Gauss-Bonnet formula imply that

$$
\sqrt{\frac{K_{j}\left(y / t_{j}\right)}{K_{j}\left(Q_{j}\right)}} e^{u_{j}} \rightarrow e^{u} \text { in } L^{2}\left(\mathbf{S}^{2}\right) .
$$

This implies that for any $\epsilon>0$, we can find a small neighborhood $B_{\delta}(-Q)$ of $-Q$ such that $\int_{B_{\delta}(-Q)} e^{2 u_{j}}<\epsilon$. Under this small integral condition, by a now standard pointwise estimate, $u_{j}$ has uniform $L^{\infty}$ bound near $-Q$, and thus uniform global $L^{\infty}$ bound on $\mathbf{S}^{2}$. Then it is easy to conclude that $u_{j} \rightarrow u$ uniformly in $C^{2}$ (subject to a selection of a subsequence). The remaining essentially repeats the proof in $[\mathrm{H}]$. The main idea is to make use of the Kazdan-Warner condition, expanding and singling out the leading terms to show that the above blow up can not happen. Note how condition (7) above comes in. When we have a sequence of $K_{j}$ to deal with, we can no longer conclude that the possible blow up point $Q$ is a critical point of the $K_{j}$ 's anymore. Instead, we can conclude that the gradient of the $K_{j}$ 's near $Q$ is small, thus the condition (7) allows the original argument to go through.

To establish Theorem A for $n=3$, we only need to establish

$$
\max _{x \in \mathbf{S}^{3}} v \leq C .
$$

Since the rest follows from standard elliptic theories.

Suppose the contrary of (9). Then for some $d>0$, there exists a sequence of $C^{2}$ functions $\left\{K_{i}\right\}$ which converge in $C^{2}$ norm to some positive function 
and a sequence $\left\{v_{i}\right\} \in C^{3}\left(\mathbf{S}^{3}\right)$ of solutions of (2) with $K$ replaced by $K_{i}$ with the following properties:

$$
\begin{gathered}
\min _{\left\{x \in \mathbf{S}^{3},\left|\nabla K_{i}(x)\right| \leq d\right\}}\left|\Delta_{g_{0}} K_{i}(x)\right| \geq d, \\
\lim _{i \rightarrow \infty} \max _{x \in S^{3}} v_{i}=\infty .
\end{gathered}
$$

Let $q_{i} \in \mathbf{S}^{3}$ be a maximum point of $v_{i}$. It is proved by $\mathrm{R}$. Schoen [Sc2] that there is precisely one isolated simple blow up point. See Definition 0.3 in [L2] for the definition of isolated simple blow up points and also a proof of the above fact under the present hypotheses. This gives strong estimates for $v_{i}$ (see Proposition 2.3 and Lemma 2.4 in [L2]). Using this information and the Kazdan-Warner identity, we reach a contradiction.

\section{ACKNOWLEDGEMENTS}

Part of this work was completed while the second author was visiting CMA of The Australian National University. He would like to thank Professor N. Trudinger and the faculty of CMA for their hospitality.

\section{REFERENCES}

[A] T. AubIN, Nonlinear analysis on manifolds. Monge-Ampère equations, SpringerVerlag, New York, 1982.

[BC] A. BAHRI and J. M. CORON, The scalar-curvature problem on standard threedimensional sphere, J. of Func. Anal., Vol. 95, 1991, pp. 106-172.

[BiE] G. BIANCHI and H. EgNell, An ODE approach to the equation $\Delta u+K u^{\frac{n+2}{n-2}}=0$, in $\mathbf{R}^{n}$, Math. Z., Vol. 210, 1992, pp. 137-166.

[BE] J. P. Bourguignon and J. P. EzIN, Scalar curvature functions in a conformal class of metric and conformal transformations, Tran. Amer. Math. Soc., Vol. 301, 1987, pp. 723-736.

[CL] K. C. ChANG and J. Q. LiU, On Nirenberg's problem, International J. of Math., Vol. 4, 1993, pp. 35-58.

[CGY] S. Y. CHANG, M. J. GURSKY and P. YANG, The scalar curvature equation on 2and 3-sphere, Calculus of Variations and Partial Differential Equations, Vol. 1, 1993, pp. 205-229.

[CY1] S. Y. CHANG and P. YANG, Conformal deformations of metrics on $\mathbf{S}^{2}, J$. Diff. Geom., Vol. 27, 1988, pp. 256-296.

[CD] W. ChEn and W. Ding, Scalar curvature on $\mathbf{S}^{n}$, Trans. Amer. Math. Soc., Vol. 303, 1987, pp. 365-382.

[ChL] W. CHEN and C. LI, A necessary and sufficient condition for the Nirenberg problem, Comm. Pure Appl. Math., Vol. 48, 1995, pp. 657-667.

[CS] K. CHENG and J. SMOLlER, Conformal metrics with prescribed Gaussian curvature on $\mathbf{S}^{2}$, Trans. Amer. Math. Soc., Vol. 336, 1993, pp. 219-255. 
[ES] J. Escobar and R. SCHOEN, Conformal metrics with prescribed scalar curvature, Invent. Math., Vol. 86, 1986, pp. 243-254.

[H] Z. C. HAN, Prescribing Gaussian curvature on $\mathbf{S}^{2}$, Duke Math. J., Vol. 61, No. 3, 1990, pp. 679-703.

[KW] J. KAZDAN and F. WARNER, Existence and conformal deformation of metrics with prescribed Gaussian and scalar curvature, Ann. of Math., Vol. 101, 1975, pp. 317-331.

[K] D. KoutroufIOTIS, Gaussian curvature and conformal mapping, J. Diff. Geom., Vol. 7, 1972, pp. 479-488.

[GT] D. Gilbarg and N. S. TRUdinger, Elliptic partial differential equations, 2nd edition, Grundlehoen der mathematischen Wissenschaften 224, Springer-Verlag, 1983.

[HV] E. Hebey and M. Vaugon, Meilleures constantes dans le théorème $\mathrm{d}^{\prime}$ inclusion de Sobolev et multiplicité pour les problèmes de Nirenberg et Yamabe, Indiana Univ. Math. J., Vol. 41, 1992, pp. 377-407.

[H] C. Hong, A best constant and the Gaussian curvature, Proc. of Amer. Math. Soc., Vol. 97, 1986, pp. 734-747.

[L1] Y. Y. LI, Prescribing scalar curvature on $\mathbf{S}^{3}, \mathbf{S}^{4}$ and related problems, J. Functional Analysis, Vol. 118, 1993, pp. 43-118.

[L2] Y. Y. LI, Prescribing scalar curvature on $\mathbf{S}^{n}$ and related problems, Part I, J. Differential Equations, Vol. 120, 1995, pp. 319-410.

[L3] Y. Y. LI, Prescribing scalar curvature on $\mathbf{S}^{n}$ and related problems, Part II: Existence and compactness, Comm. Pure Appl. Math., Vol. 49, 1996.

[M] J. Moser, On a nonlinear problem in differential geometry, Dynamical systems (M. Peixoto, ed.) Academic Press, New York, 1973.

[Sc1] R. SchoEN, Variational theory for the total scalar curvature functional for Riemannian metrics and related topics, in Topics in Calculus of Variations, Lecture notes in mathematics, No. 1365, edited by M. Giaquinta, SpringerVerlag, 1989, pp. 120-154.

[Sc2] R. Schoen, Private notes of special topics in geometry courses in Stanford University and New York University, 1988 and 1989.

[XY] X. XU and P. YANG, Remarks on prescribing Gauss curvature, Trans. Amer. Math. Soc., Vol. 336, 1993, pp. 831-840.

[Z] D. Zhang, New results on geometric variational problems, thesis, Stanford University, 1990.

(Manuscript received August 5, 1994.) 